\title{
Exceptional points in quantum and classical dynamics
}

\author{
A V Smilga ${ }^{1}$ \\ SUBATECH, Université de Nantes, 4 rue Alfred Kastler, BP 20722, Nantes 44307, France \\ E-mail: smilga@subatech.in2p3.fr
}

Received 4 November 2008, in final form 12 December 2008

Published 4 February 2009

Online at stacks.iop.org/JPhysA/42/095301

\begin{abstract}
We note that when a quantum system involves exceptional points, i.e. the special values of parameters where the Hamiltonian loses its self-adjointness and acquires the Jordan block structure, the corresponding classical system also exhibits singular behaviour associated with the restructuring of classical trajectories. A system with the crypto-Hermitian Hamiltonian $H=\left(p^{2}+\right.$ $\left.z^{2}\right) / 2-\mathrm{i} g z^{5}$ and hyper-elliptic classical dynamics is studied in detail. Analogies with supersymmetric Yang-Mills dynamics are elucidated.
\end{abstract}

PACS numbers: $03.65 .-\mathrm{w}, 12.60 . \mathrm{Jv}$

\section{Introduction}

A sufficient condition for the spectrum of a Hamiltonian to be real is its self-adjointness, $H^{\dagger}=H$. However, there exists a rich class of systems whose Hamiltonian is not manifestly Hermitian, but the spectrum is still real. Probably, the simplest such example is the matrix Hamiltonian:

$$
H=\left(\begin{array}{ll}
1 & 1 \\
0 & 2
\end{array}\right)
$$

with two real eigenvalues $\lambda_{1}=1$ and $\lambda_{2}=2$ respectively. Such systems have been intermittently discussed since the mid-seventies [1], but interest in this problem was considerably boosted after paper [2] appeared, where systems with complex potentials $V(x)=x^{2}(\mathrm{i} x)^{\epsilon}$ were studied, and it was shown that the spectrum of the corresponding Hamiltonians is real.

Generically, a Hamiltonian involving only real eigenvalues can be transformed in a manifestly Hermitian form by a similarity transformation [3], $H \rightarrow R H R^{-1}$. If $H$ is not manifestly Hermitian, $R$ is not unitary. This amounts to modifying the Hilbert space measure so that the probability $P=\langle\psi|M| \psi\rangle$ defined with the new measure $M$ is conserved and the

1 On leave of absence from ITEP, Moscow, Russia. 
theory is unitary ${ }^{2}$. One can call a Hamiltonian of this type crypto-Hermitian (Hermitian in disguise) $[4,5]$.

However, not all Hamiltonians with real eigenvalues are crypto-Hermitian. In some cases, a Hamiltonian cannot be rendered Hermitian (and eventually diagonalized) by a similarity transformation. The simplest example is a $2 \times 2$ matrix representing a Jordan block, $H=\left(\begin{array}{ll}1 & 1 \\ 0 & 1\end{array}\right)$. The exceptional points when this happens are associated with the degeneracy of eigenvalues [6]. Exceptional points have measure zero in the space of parameters.

There are also exceptional points involving multidimensional Jordan blocks. For a higher derivative Pais-Uhlenbeck oscillator [7] at equal frequencies, the dimension of emerging Jordan blocks is even infinite [8,9]. A more typical kind of an exceptional point is when only a couple of eigenvalues coalesce. By changing the parameters beyond the exceptional point, a pair of complex eigenvalues that are conjugate to each other appears ${ }^{3}$.

A numerical study performed in [2] displayed an infinite number of exceptional points in the parameter $\epsilon$ for the Hamiltonian

$$
H=p^{2}+z^{2}(\mathrm{i} z)^{\epsilon} .
$$

The exceptional points lie in the interval $\epsilon \in(-1,0)$. The leftmost exceptional point is $\epsilon_{*} \approx-0.578$. When $\epsilon<\epsilon_{*}$, the spectrum of the Hamiltonian involves only one real eigenvalue (the ground state), with all other eigenvalues coming in complex conjugate pairs. At $\epsilon=\epsilon_{*}$, one of such pairs coalesces and, at still larger $\epsilon$, is transformed into a pair of close real eigenvalues. Then comes the turn of the second pair. The infinite number of exceptional points has an accumulation point at $\epsilon=0$ [10]. At $\epsilon>0$, complex eigenvalues disappear and the Hamiltonian is crypto-Hermitian.

A similar phenomenon was observed in [11] for the Hamiltonian

$$
H=\frac{p^{2}+z^{2}}{2}-\mathrm{i} g z^{5}
$$

There are two different spectral problems corresponding to this Hamiltonian (see $[11,12]$ for detailed explanations). One of the problems is defined in the Stokes wedges:

$$
\begin{aligned}
-\frac{\pi}{14} & <\operatorname{Arg}(z)<\frac{3 \pi}{14}, \\
\frac{11 \pi}{14}<\operatorname{Arg}(z) & <\frac{15 \pi}{14},
\end{aligned}
$$

including the real axis. It turns out that the spectrum is real there for all values of $g$. But for another spectral problem defined in the wedges

$$
\begin{aligned}
& -\frac{5 \pi}{14}<\operatorname{Arg}(z)<-\frac{\pi}{14} \\
& \frac{15 \pi}{14}<\operatorname{Arg}(z)<\frac{19 \pi}{14}
\end{aligned}
$$

in the complex $z$-plane, the situation is different. The spectrum is real (and the Hamiltonian is crypto-Hermitian) for large enough $g$, but the system involves an infinite number of

2 The explicit forms of $R$ and $M$ for the Hamiltonian (1) are

$$
R=\left(\begin{array}{cc}
1 & -1 \\
0 & 1
\end{array}\right), \quad M=R^{T} R=\left(\begin{array}{cc}
1 & -1 \\
-1 & 2
\end{array}\right)
$$

3 Again, the simplest example is the matrix $H=\left(\begin{array}{ll}1 & 1 \\ \alpha & 1\end{array}\right)$. When $\alpha$ is small and positive, we have a pair of close real eigenvalues. When $\alpha$ is small and negative, there is a pair of complex conjugate eigenvalues. $\alpha=0$ is the exceptional point. 
exceptional points at small $g$. If $g<g_{*} \approx 0.037$, a pair of complex eigenvalues appears. If $g<g_{* *} \approx 0.007$, there are at least two such pairs, etc.

A question that can be asked is: whether the presence of these quantum exceptional points displays themselves in some way also in the dynamics of the corresponding classical systems? A partial answer to this question was obtained in [13] where a series of critical values of the parameter $\epsilon$, where the pattern of classical trajectories in the problem (3) is changed, was found. The values of the classical exceptional points do not coincide with the values of the quantum exceptional points. One can only say that the presence of the former is associated with the presence of the latter.

The problem (3) is rather complicated, however. The Riemann surface for the potential $\sim z^{2+\epsilon}$ has generically an infinite number of sheets; the classical trajectories can visit a number of these sheets and look complicated. That is why we decided to study this question for the system (4), which is much simpler. The classical trajectories represent in this case hyperelliptic functions known to mathematicians [14].

The result is as follows. There are no classical exceptional points for the system (4) with a positive energy. When the energy is negative, there is a single classical exceptional point:

$$
g_{*}^{\text {class }}=\frac{1}{5}\left(-\frac{3}{10 E}\right)^{3 / 2} \approx \frac{0.0329}{|E|^{3 / 2}} .
$$

As was mentioned above, a classical exceptional point is the point where the pattern of the classical trajectories changes. For the system (4), the reason for this change is very simple: it happens that at $g=g_{*}^{\text {class }}$ two of the five turning points, i.e. two of the five solutions to the equation

$$
V(x)=\frac{z^{2}}{2}-\mathrm{i} g z^{5}=E<0,
$$

coalesce. This phenomenon has a lot in common with the so-called Argyres-Douglas phenomenon in supersymmetric Yang-Mills theory [15]). We will dwell upon this issue in section 4 .

Before discussing the dynamics of the system (4), we want to make few comments on the classical dynamics of a simpler system:

$$
H=\frac{p^{2}+\omega^{2} z^{2}}{2}+\mathrm{i} g z^{3}
$$

where exceptional points do not appear at either the classical or the quantum level.

\section{Complex cubic potential and elliptic dynamics}

Consider the system (9). Complex classical trajectories are the solutions to the equation

$$
\frac{\dot{z}^{2}}{2}+\frac{\omega^{2} z^{2}}{2}+\mathrm{i} g z^{3}=E
$$

The solutions to this equation with real positive energies (especially, in the case $\omega=0$ ) were found numerically in [2]. The solutions with real negative energies were found in [11]. A simple remark that we want to make here is that the solution to equation (10) has a name-it is the Weierstrass function. Let us for simplicity set $\omega=0, g=1$. The equation is brought into the canonical Weierstrass form [16],

$$
\dot{y}^{2}=4 y^{3}-g_{2} y-g_{3},
$$

with $g_{2}=0, g_{3}=E / 2$, by setting $z=2 \mathrm{i} y$. 


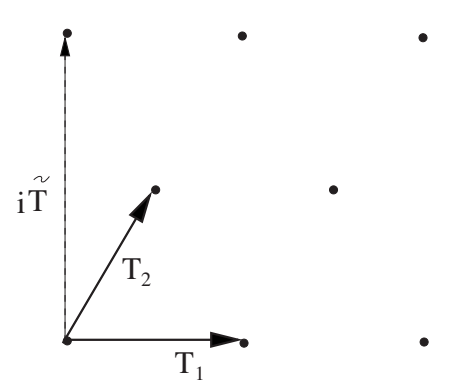

Figure 1. Periods of the Weierstrass function.

The Weierstrass function $z=P\left(t, g_{2}, g_{3}\right)$ is an elliptic double-periodic complex function. Generically, the periods are complex, but in our case one of the periods is real. For positive energies,

$$
T_{1}=5 \sqrt{\frac{\pi}{6}} \frac{\Gamma(4 / 3)}{\Gamma(11 / 6)} E^{-1 / 6} \approx 3.434 E^{-1 / 6}
$$

Another period is still complex, $T_{2}=T_{1} \mathrm{e}^{\mathrm{i} \pi / 3} . T_{2}$ is obtained from $T_{1}$ by rotation in the complex $E$ plane, $E \rightarrow E \mathrm{e}^{-2 \mathrm{i} \pi}$. Any linear combination of $T_{1}$ and $T_{2}$ with integer coefficients is also a period. A purely imaginary combination $\mathrm{i} \tilde{T}=2 T_{2}-T_{1}=\mathrm{i} \sqrt{3} T_{1}$ represents a particular interest. The physical interpretation of the periods $T_{1}$ and $\tilde{T}$ is clear [2, 11]. A periodicity with respect to the real time shift $t \rightarrow t+T_{1}$ is a usual physical periodicity of the trajectories. For all trajectories, the period is the same. The imaginary time shift transforms one trajectory into another ${ }^{4}$.

One can also be interested in the solutions to equation (10) at complex $E$. It is still a Weierstrass function, but all its periods are now complex. This means that the solution is not periodic in real physical time. Such solutions were studied numerically in [17].

The family of all trajectories contains two distinguished members that can be called stem trajectories. One of the stem trajectories connects two turning points (solutions to the equation $\left.V(z)=\mathrm{i} z^{3}=E\right)$ in the lower half-plane: $z_{1}=E^{1 / 3} \mathrm{e}^{-5 \mathrm{i} \pi / 6}$ and $z_{2}=E^{1 / 3} \mathrm{e}^{-\mathrm{i} \pi / 6}$. Another stem trajectory goes from the turning point $z_{3}=\mathrm{i} E^{1 / 3}$ to infinity. The shift $t \rightarrow t+\mathrm{i} \tilde{T} / 4$ transforms one stem trajectory into another. The shift $t \rightarrow t+\mathrm{i} \tilde{T} / 2$ transforms the function $z=2 \mathrm{i} P(t, 0, E / 2)$ to $z=2 \mathrm{i} P(-t, 0, E / 2)$ and the shift by $\mathrm{i} \tilde{T}$ leaves the Weierstrass function intact. This is all illustrated in figures 1 and 2 .

Translating these observations into a standard mathematical language, one can say that the Weierstrass function describes the motion over the Riemann surface of the function $\sqrt{E-\mathrm{i} z^{3}}$. The turning points and infinity are nothing but the branching points of this function. This Riemann surface has the topology of torus. Two periods $T_{1,2}$ correspond to two cycles of this torus.

One can also plot the imaginary time trajectories $2 \mathrm{i} P(a+\mathrm{i} \tilde{t}, 0,1 / 2), \tilde{t} \in(0, \tilde{T})$, and observe by inspecting equation (11) that they coincide up to a sign with the real time trajectories $2 \mathrm{i} P(\tilde{t}-\mathrm{i} a, 0,-1 / 2)$ having negative energies and studied in [11].

4 This transformation can be interpreted as a gauge transformation [11], but it is not a point of interest for us now. 


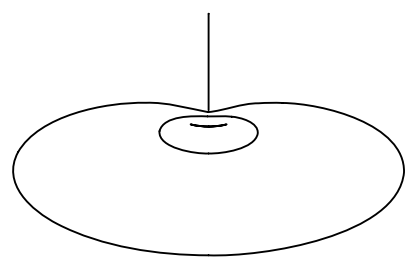

Figure 2. The trajectories $z=2 \mathrm{i} P(t+\mathrm{i} a, 0,1 / 2), t \in\left(0, T_{1}\right)$. The vertical line $(a=0)$ is the stem trajectory connecting the branching points $z=\mathrm{i}$ and $z=\infty$. The 'smile' $(a=\tilde{T} / 4)$ is the stem trajectory connecting the branching points $z=\mathrm{e}^{-5 \mathrm{i} \pi / 6}$ and $z=\mathrm{e}^{-\mathrm{i} \pi / 6}$. The trajectories with intermediate values $a=\tilde{T} / 16$ and $a=\tilde{T} / 8$ are also plotted.

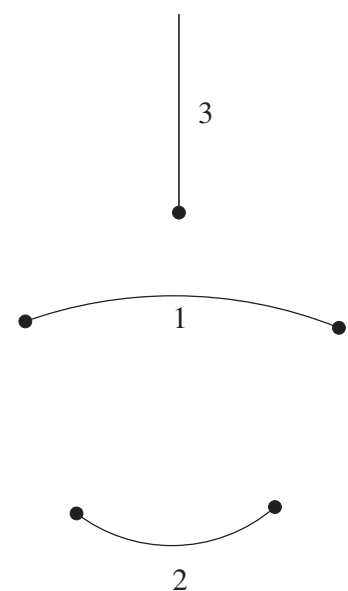

Figure 3. Stem trajectories for the potential $-\mathrm{i} z^{5}$.

\section{The potential $V(z)=z^{2} / 2-\mathrm{i} g z^{5}$ and hyper-elliptic dynamics}

Classical complex trajectories for the Hamiltonian (4) have much in common with the elliptic trajectories of the previous section. They are hyper-elliptic trajectories describing the motion over the Riemann surface of the function $\sqrt{E-V(z)}$. This Riemann surface has genus 2 and two pairs of cycles. The latter implies that the equation

$$
\frac{\dot{z}^{2}}{2}+V(z)=E
$$

has now different types of solutions stemming from the trajectories that connect three different pairs of branching points of the function $\sqrt{E-V(z)}$. First, let the energy be positive and $V(z)=-\mathrm{i} z^{5}$ (without the quadratic term). The branching points are in the vertices of the pentagon and at infinity. Three different stem trajectories are depicted in figure 3 . The corresponding periods are $[2,11]$

$$
\begin{aligned}
& T_{1}=\frac{7}{5} \sqrt{2 \pi} \cos \frac{\pi}{10} \frac{\Gamma(6 / 5)}{\Gamma(17 / 10)} E^{-3 / 10} \\
& T_{2}=\frac{7}{5} \sqrt{2 \pi} \cos \frac{3 \pi}{10} \frac{\Gamma(6 / 5)}{\Gamma(17 / 10)} E^{-3 / 10},
\end{aligned}
$$

and $T_{3}=T_{1}-T_{2}$.

Similar to what we had for the cubic potential, the solutions to equation (13) are also periodic with respect to imaginary time shifts: 


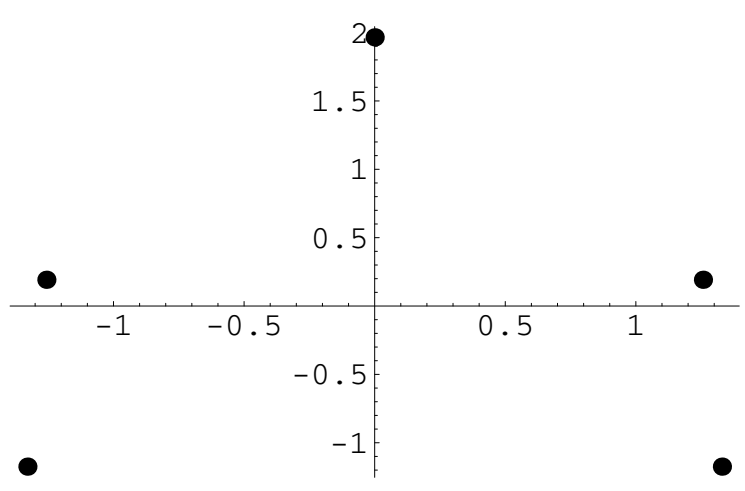

Figure 4. Turning points. $E=1, g=0.1$.

$$
\begin{aligned}
& \mathrm{i} \tilde{T}_{1}=\mathrm{i} \frac{7}{5} \sqrt{2 \pi}\left(1+2 \sin \frac{3 \pi}{10}+\sin \frac{\pi}{10}\right) \frac{\Gamma(6 / 5)}{\Gamma(17 / 10)} E^{-3 / 10} \\
& \mathrm{i} \tilde{T}_{2}=\mathrm{i} \frac{7}{5} \sqrt{2 \pi}\left(1+\sin \frac{3 \pi}{10}\right) \frac{\Gamma(6 / 5)}{\Gamma(17 / 10)} E^{-3 / 10} \\
& \mathrm{i} \tilde{T}_{3}=\mathrm{i} \frac{7}{5} \sqrt{2 \pi}\left(\sin \frac{3 \pi}{10}+\sin \frac{\pi}{10}\right) \frac{\Gamma(6 / 5)}{\Gamma(17 / 10)} E^{-3 / 10} .
\end{aligned}
$$

The periods $(14,15)$ are all inter-related under rotations in the complex $E$ plane (the monodromies $)^{5}$. For example,

$$
T_{1}\left(E \mathrm{e}^{-2 \mathrm{i} \pi}\right)=\frac{\mathrm{i} \tilde{T}_{2}-T_{2}}{2}, \quad T_{2}\left(E \mathrm{e}^{-2 \mathrm{i} \pi}\right)=\frac{\mathrm{i} \tilde{T}_{3}-T_{3}}{2} .
$$

This leads to three families of trajectories with positive energies and three families of trajectories with negative energies ${ }^{6}$.

Let us now switch on the quadratic term and investigate how the pattern of trajectories is changed when changing $g$. First, let the energy be positive. Note that for all $g$, equation (8) still has five distinct roots (see figure 4).

We see that the turning points are still symmetric with respect to the imaginary axis. There are still three families of trajectories stemming from the trajectories that have the same qualitative pattern as in figure 3. Nothing changes essentially when $g$ is changed.

When $E<0$, the situation is different. There is a point (7) where two of the roots of equation (8) coalesce. The root patterns slightly above the exceptional point $g=g^{*}$ and slightly below it are shown in figure 5 .

When $g>g^{*}$, there are three families of orbits. Their stem trajectories are shown in figure $6(a)$. When $g<g *$, the pattern of the stem trajectories is different (see figure $6(b)$ ). One can note that the stem trajectory connecting the low pair of the turning points with $\operatorname{Re}(x) \neq 0$ exists at both $g>g^{*}$ and $g<g^{*}$, but its form is different. When $g \rightarrow g^{*}+\mathrm{i} 0$, the trajectory crosses itself, after which the 'appendix' going around the low turning point at the imaginary axis disappears.

\footnotetext{
5 See Proposition 7 in [18].

6 To be more precise, the third family is degenerate in this case and consists of only one member: the stem trajectory starting at $z=\mathrm{i} E^{1 / 5}$ and going away to $z=\mathrm{i} \infty$ in finite time, $t_{\text {escape }}=T_{3} / 2$.
} 


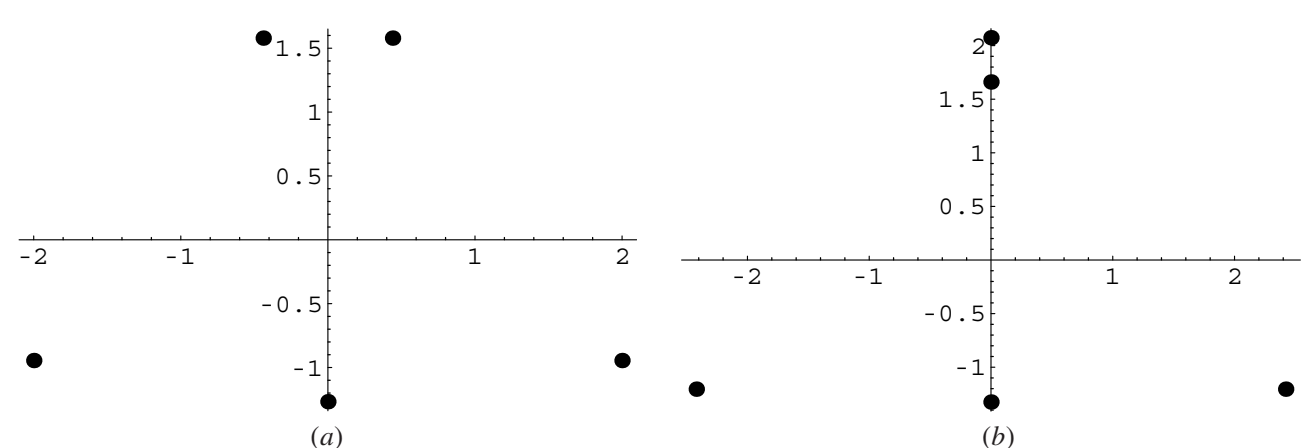

Figure 5. Turning points. $E=-1 ;(a) g=0.06,(b) g=0.03$.

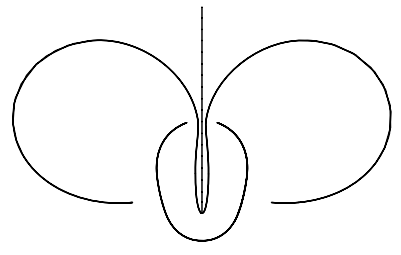

(a)

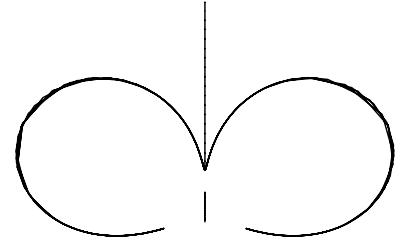

(b)

Figure 6. Stem trajectories. $E=-1$; (a) $g=0.06,(b) g=0.01$. The small gaps between the vertical lines going to $\mathrm{i} \infty$ and the curves below are indistinguishable.

\section{Analogies with SYM}

As was already mentioned, the restructuring of classical trajectories observed in this paper has much in common with the Argyres-Douglas phenomenon that occurs in SYM theory. Let us briefly explain this point here (see [19] for more details).

$\mathcal{N}=2$ supersymmetric gauge theories are known to include vacuum moduli spaces: valleys of degenerate classical vacua parametrized by vacuum expectation values of scalar fields [20]. These valleys have some distinguished singular points where the mass of certain objects present in the theory (the monopoles and dyons) vanishes. The original AD observation was that, in some theories and for certain values of parameters, the moduli space singularities might coalesce.

An analogy between this phenomenon and the classical exceptional point (7) exists, but is not so manifest. It becomes much more clear for the theories where $\mathcal{N}=2$ is broken down to $\mathcal{N}=1$. The continuous degeneracy of the vacuum valleys is lifted in this case, and we are left with a finite number of different classical vacua. These vacua can be separated by domain walls, the classical solutions to the equations of motion with proper boundary conditions. Mathematically, domain walls interpolating between the classical vacua play exactly the same role as the stem trajectories interpolating between the turning points in the QM models discussed above.

The point is that, in some $\mathcal{N}=1$ models, the parameters can be chosen such that the classical vacua coalesce. This phenomenon is akin to the original AD phenomenon (merging of singularities in vacuum moduli spaces). One can call it the AD phenomenon of the second kind. It was first discovered for a somewhat exotic model based on the $G_{2}$ gauge group and 
involving besides $\mathcal{N}=1$ gauge supermultiplet three different chiral multiplets $S_{\alpha=1, \ldots, 7}^{i=1,2,3}$ in the fundamental representation of $G_{2}$ [21]. The superpotential of the model is

$$
W=-\frac{m}{2}\left(S_{\alpha}^{i}\right)^{2}-\frac{\lambda}{6} \mathrm{e}_{i j k} f^{\alpha \beta \gamma} S_{\alpha}^{i} S_{\beta}^{j} S_{\gamma}^{k},
$$

where $f^{\alpha \beta \gamma}$ is the invariant antisymmetric tensor of $G_{2}{ }^{7}$. To analyse the vacuum structure, one has to add to the superpotential (17) a nonperturbative instanton-generated superpotential of the Affleck-Dine-Seiberg type [22]. In this case, it has the form

$$
\mathcal{W}^{\text {inst }}=\frac{\Lambda^{9}}{\mathcal{B}^{2}-\operatorname{det} \mathcal{M}},
$$

where $\mathcal{B}$ and $\mathcal{M}$ are the gauge-invariant moduli:

$$
\mathcal{M}^{i j}=S_{\alpha}^{i} S_{\alpha}^{j}, \quad \mathcal{B}=\frac{1}{6} \epsilon_{i j k} S_{\alpha}^{i} S_{\beta}^{j} S_{\gamma}^{k}
$$

and $\Lambda$ is the confinement scale.

Adding the superpotentials (17) and (18), we obtain a theory with two dimensionless parameters: the Yukawa coupling $\lambda$ and the ratio mass $m / \Lambda$ respectively. The equation determining the chirally asymmetric classical vacua ${ }^{8}$ is of the sixth order:

$$
\frac{\partial \mathcal{W}}{\partial(\text { moduli })}=0 \longrightarrow m u^{4}\left(1-\frac{\lambda^{2} u}{m^{2}}\right)^{2}=\Lambda^{9}
$$

where $u=\frac{1}{3} \operatorname{Tr} \mathcal{M}$. Generically, there are six distinct solutions. However, when $\lambda^{2} \approx$ $0.385 \mathrm{~m}^{2} / \Lambda^{2}$, two of these vacua coalesce.

The simplest model where the AD phenomenon of the second kind occurs is based on the $S U$ (2) gauge group and involves a massive adjoint chiral multiplet and a pair of fundamental chiral multiplets. This model obtained by a deformation of $\mathcal{N}=2$ supersymmetric QCD (the model involving besides the gauge $\mathcal{N}=2$ multiplet also a matter hypermultiplet) and analysed in [24] involves generically three vacua, but, for some values of masses and Yukawa couplings, two of them coalesce.

When the vacua merge, the domain wall interpolating between them disappears. The analogy with the classical exceptional point (7) is obvious.

\section{Acknowledgments}

I acknowledge numerous illuminating discussions with A Gorsky and warm hospitality at the AEI institute in Golm where this work was finished.

\section{References}

[1] Bronzan J B, Shapiro J A and Sugar R L 1976 Phys. Rev. D 14618 Amati D et al 1976 Nucl. Phys. B 112107

Gasymov M G 1980 Funct. Anal. Appl. 1411

Calicetti E, Graffi S and Maioli M 1980 Comm. Math. Phys. 7551

Scholtz F G, Geyer H B and Hahne F J W 1992 Ann. Phys., NY 21374

[2] Bender C M and Boettcher S 1998 Phys. Rev. Lett. 805243

[3] Mostafazadeh A 2002 J. Math. Phys. 43 2814; 3944

Mostafazadeh A 2005 J. Phys. A: Math. Gen. 386557

[4] Feinberg J and Zee A 1999 Phys. Rev. E 596433

7 Note that $G_{2}$ can be defined as a subgroup of $S O(7)$ leaving invariant the form $f^{\alpha \beta \gamma} A_{\alpha} B_{\beta} C_{\gamma}$ for any set of three different 7-vectors $A, B, C$.

8 We leave aside a controversial issue of possible existence of the extra chirally symmetric vacuum [23]. 
[5] Ivanov E and Smilga A 2007 J. High Energy Phys. JHEP07(2007)036

[6] Heiss W D 2004 J. Phys. A: Math. Gen. 372455 and references therein

[7] Pais A and Uhlenbeck G E 1950 Phys. Rev. 79145

[8] Smilga A V 2006 Phys. Lett. B 632433

[9] Smilga A V 2008 arXiv: 0808.0139 [quant-ph]

[10] Dorey P, Millican-Slater A and Tateo R 2005 J. Phys. A: Math. Gen. 381305 (arXiv:hep-th/0410013)

[11] Smilga A 2008 J. Phys. A: Math. Theor. 41244026 (arXiv:0706.4064)

[12] Dorey P, Dunning C and Tateo R 2001 J. Phys. A: Math. Gen. 345679 Shin K C 2005 J. Math. Phys. 46082110

[13] Bender C M, Chen J-H, Darg D W and Milton K A 2006 J. Phys. A: Math. Gen. 394219

[14] Siegel C L 1988 Topics in Complex Function Theory, Vol. 2: Automorphic Functions and Abelian Integrals (New York: Wiley)

[15] Argyres P C and Douglas M R 1995 Nucl. Phys. B 44893

[16] Whittaker E T and Watson G N 1963 A Course of Modern Analysis (Cambridge: Cambridge University Press) Gradstein I S and Ryzhik I M 1965 Tables of Integrals, Series, and Products (New York: Academic)

[17] Bender C M, Brody D C and Hook D W 2008 J. Phys. A: Math. Theor. 41352003

[18] Fedorov Yu N and Gomez-Ullate D 2007 Physica D 227120 (arXiv:nlin/0607028)

[19] Gorsky A S and Smilga A V in preparation

[20] Seiberg N and Witten E 1994 Nucl. Phys. B 42619 Seiberg N and Witten E 1994 Nucl. Phys. B 431484

[21] Smilga A V 1998 Phys. Rev. D 58105014

[22] Affleck I, Dine M and Seiberg N 1984 Phys. Lett. B 137187

[23] Kovner A and Shifman M A 1997 Phys. Rev. D 562396

[24] Gorsky A, Vainshtein A and Yung A 2000 Nucl. Phys. B 584197 